\title{
ELECTRICAL DEVICE CONTROL SYSTEM THROUGH WI-FI TECHNOLOGY
}

\author{
E.M. DIACONU \\ Valahia University of Targoviste, Faculty of Electrical Engineering, Electronics and Information Technology \\ E-mail: emy_diaconu@yahoo.com
}

\begin{abstract}
The study aims for the development of a system that allows the management and control of an electrical device, this paper is an introduction to the world of smart home / IoT and transforms non-smart equipment offering the user greater control and customizable options just by connecting the desired device to the control system. The system consists of a control board that operates two relays, the relays have the main function of opening and closing the electrical circuit. The control board consists of an AMS1117 3.3V voltage lowering module, ESP8266 microcontroller and connectors for power supply and relay control. For the software part, the IDE programming language for the Arduino microcontroller was used, being an easy to use/understand and very versatile programming language.
\end{abstract}

Keywords: IoT, ESP8266, Smart

\section{INTRODUCTION}

The concept of Internet of Things (IoT) defines a world in which all automobiles, home hardware appliances, illumination systems, mobile devices, etc.) are connected all together through an Internet connection. The existence of Internet of Things (IoT) doesn't need computers. Each device that is equipped with the necessary electronic hardware can take part of the IoT universe. The electronic hardware can vary depending on what task the user wants to accomplish from the device and the hardware usually can be grouped in two basic categories:

- the device must take data from the surrounding environment via sensors.

- the device must be connected to the Internet for data transfer.

Therefore, the main hardware needed from the device to achieve IoT status are sensors and the ability to connect to the Internet.

As claimed by the analysts, in the year 2015 there were from 10 to 20 billion devices that were connected via an Internet connection. All the connected devices formed the foundation for the IoT universe. The number of connected objects in 2015 was small compared to how many will be connected in 2021. Estimates vary, but it is generally predicted that the number of the devices that will be connected by 2021 will reach about $40-50$ billion in number, from pens to housing, machinery and industrial equipment. In Figure 1 we can see the graphical representation that shows all the devices that can be connected together in the IoT universe.

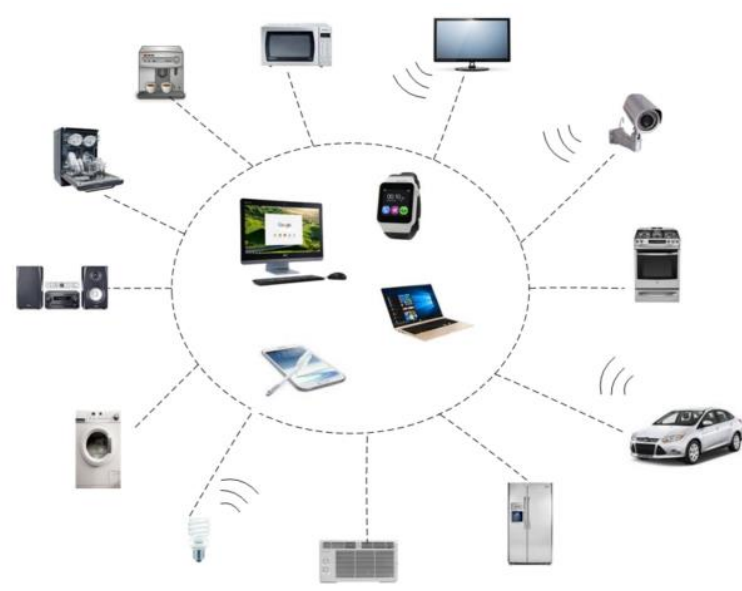

Figure 1. IoT devices.

The Internet of Things universe has become one of the most challenging research topics in the field of ICT (information and communication technology). The Internet of Things offers an amazing number of business opportunities, many of which are known only to experts in the field. In general, the media focuses on the consumer segment of the Internet of Things. There is no doubt that consumer products have an important place in the universe of the Internet of Things but the businesses that are not involved in that market may believe that the Internet of Things has nothing to offer. However, IoT will have a big influence on all the levels of business operations, despite the type of industry. The problems that companies have been facing for decades will diminish significantly and, in many cases, disappear.

According to a study by RAND Europe, the annual global economic potential of the Internet of Things in all targeted sectors ranges from 1.39 trillion USD (about 1.08 trillion EURO) in 2015 to 13.9 trillion USD (about 10.9 trillion EURO) in 2021, approximately, the European Union GDP. By 2021, the Internet of Things will no longer be an niche segment, the number of industrial activities that will not be influenced by the Internet of Things will be insignificant. Even in 2015, there were few industries that did not use the Internet of Things at all in their activities and processes. As a result, the number of industries in which the Internet of Things is indispensable for the conduct of operations is growing.

The Gartner company has estimated that by 2021 , there will exist about 27 billion devices connected together in the IoT universe. According to a study made by Pew Research Center, 83 percent of technology experts and 
Internet users acknowledged the idea that IoT, with integrated and portable computer systems will have widespread benefits by 2025 .

\subsection{ESP8266}

ESP8266 is a economic microchip with integrated Wi-Fi function that has full TCP / IP capabilities and can be used as a microcontroller. The 8266 chips entered the market in 2014 with the ESP-01 (Figure 2) developing board/module. This module can allow other developing boards to connect to a Wi-Fi network. So far, they have been given the generic name of ESP-xx modules, with $\mathrm{xx}$ $=01.02, \ldots 12,13.14$. Many of the ESP-xx modules include an LED that can be set from the program to flash, this function is useful to indicate the activity of the chip. The user can choose from several antenna that are made for the ESP-xx boards, including a tracking antenna, a ceramic antenna, and an external antenna. Earlier ESP-xx models are not included with an USB-to-UART convertor and there is no Micro-USB connector (required to provide host connectivity and board power, respectively). For previous ESP-xx modules, these two elements (the USBSerial adapter and a 3.3-volt controller) had to be purchased separately.

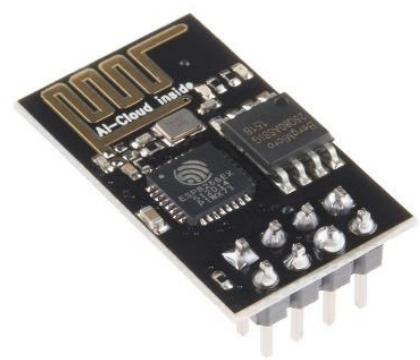

Figure 2. ESP-01 module.

The ESP-01 module comes with integrated SPI, UART, TCP/IP protocol stack, PLL, regulators, power management units, temperature sensor, 32-bit CPU and supports antenna diversity. The ESP-01 has the next specifications:

- Operating voltage: $3.3 \mathrm{~V}$

- GPIO pins: 2

- Power consumption in standby: $<1.0 \mathrm{~mW}$

- $\quad 802.11 \mathrm{~b} / \mathrm{g} / \mathrm{n}$ protocol

- $\quad$ Supports AP, STA, AP + STA mode

- $\quad$ Power output of $+19.5 \mathrm{dBm}$ in $802.11 \mathrm{~b}$ mode

- PCB dimensions: 11 x $10 \mathrm{~mm}$

\subsection{AMS1117}

The AMS1117-3.3 voltage lowering module (Figure 3 ) is used for lowering the voltage from $10-4.2 \mathrm{~V}$ range to $3.3 \mathrm{~V}$ thus offering the voltage needed to supply components that require a voltage of $3.3 \mathrm{VDC}$ for operation like the ESP-01 module. The AMS1117 specifications are:

- Input voltage: $4.2 \mathrm{~V}-10 \mathrm{~V}$

- Output voltage: $3.3 \mathrm{~V}$
- $\quad$ Dimensions: 8.6 x $12.3 \mathrm{~mm}$.

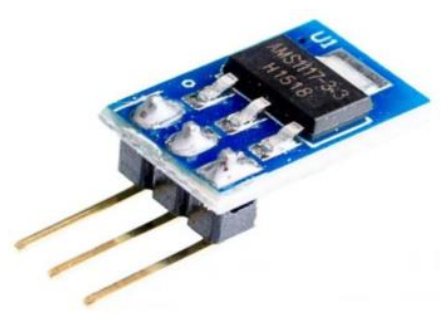

Figure 3. AMS1117 voltage lowering module.

\subsection{AC - DC Convertor}

The A.C to D.C convertor (Figure 4) is used to power all the systems components from a normal $220 \mathrm{~V}$ power outlet. The output power of the convertors is $3.5 \mathrm{~W}(5 \mathrm{~V} / 700$ $\mathrm{mAh})$. The convertor has the next specifications:

- Input voltage: 85 - 220VAC, $50 \mathrm{~Hz}$

- Output voltage: 5VDC (+-0.2V)

- Power: $3.5 \mathrm{~W}$

- Output current: 700mAh

- Dimensions: 30 x $22 \mathrm{~mm}$

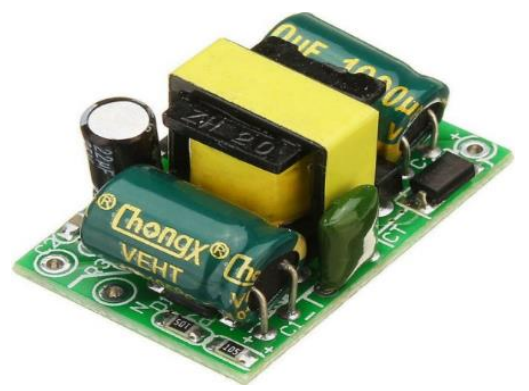

Figure 4. AC - DC Convertor.

\subsection{Relay Module}

The relay module (Figure 5) is used to control the devices by opening/closing the electrical circuit. The relay module is powered by the A.C-D.C convertor and controlled by the ESP-01 module. The relay module has the next specifications:

- Dimensions: 49 X 38 X 17 mm

- Weight: $31 \mathrm{~g}$

- Input voltage: 5V

- Number of channels: 2

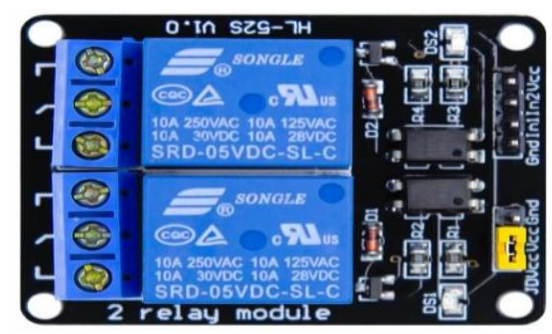

Figure 5. Relay Module. 


\section{THE CONTROL BOARD}

In Figure 6 we can see the front/backside of the control board. The control board is made from the ESP-01 socket (1), AMS1117 module (2), power connector (3) and relay module power/control connector (4).

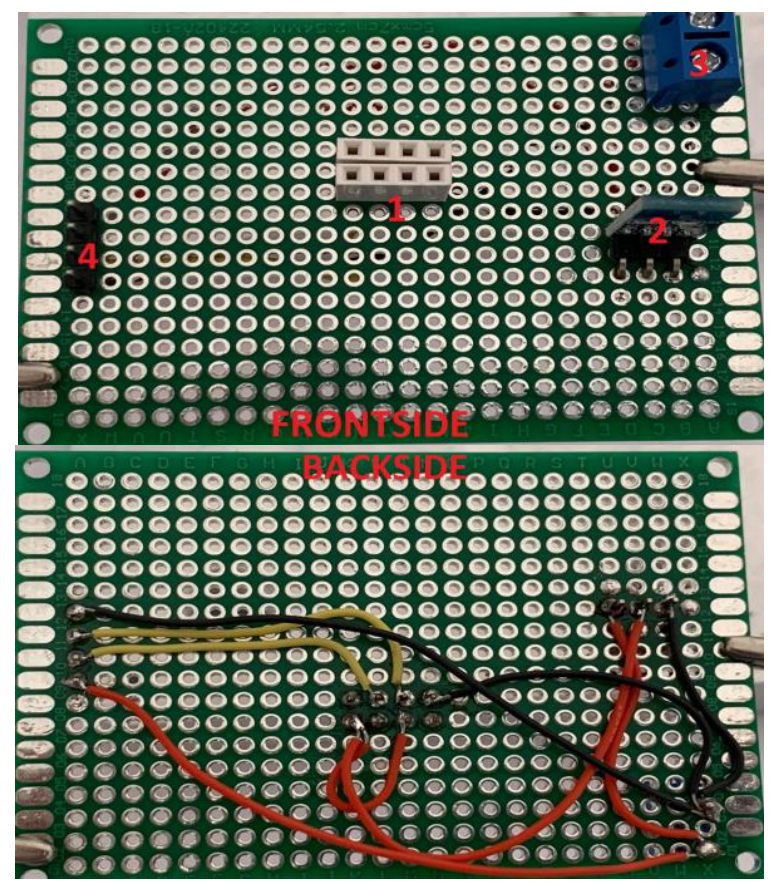

Figure 6. Control Board.

\section{THE FUNCTIONING PRINCIPALE}

The system has the ability to control electric devices via Wi-Fi through a web interface created in HTML by switching on and off the relay module thus connecting/disconnecting the electric power supply of the connected devices, the system supports 2 devices connected simultaneously. In Figure 7 we have the block diagram of the system.

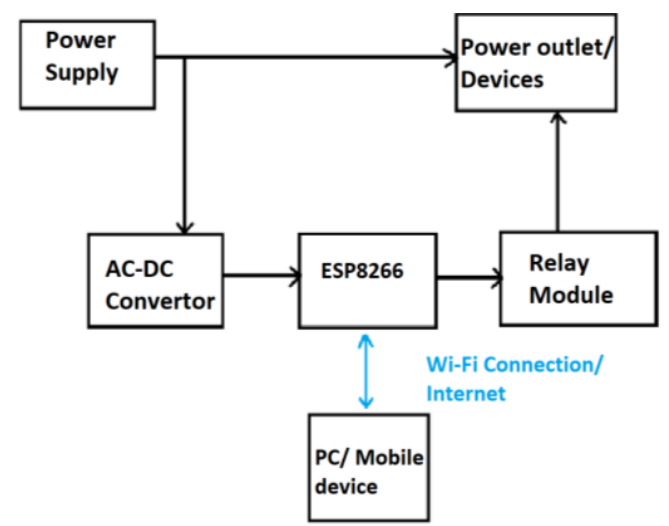

Figure 7. Block Diagram.

In Figure 8 we have the system fully assembled. The system is made from the ESP8266 control board (1), relay module 2, A.C - D.C convertor, power block (4) and the power outlets $(5,6)$.

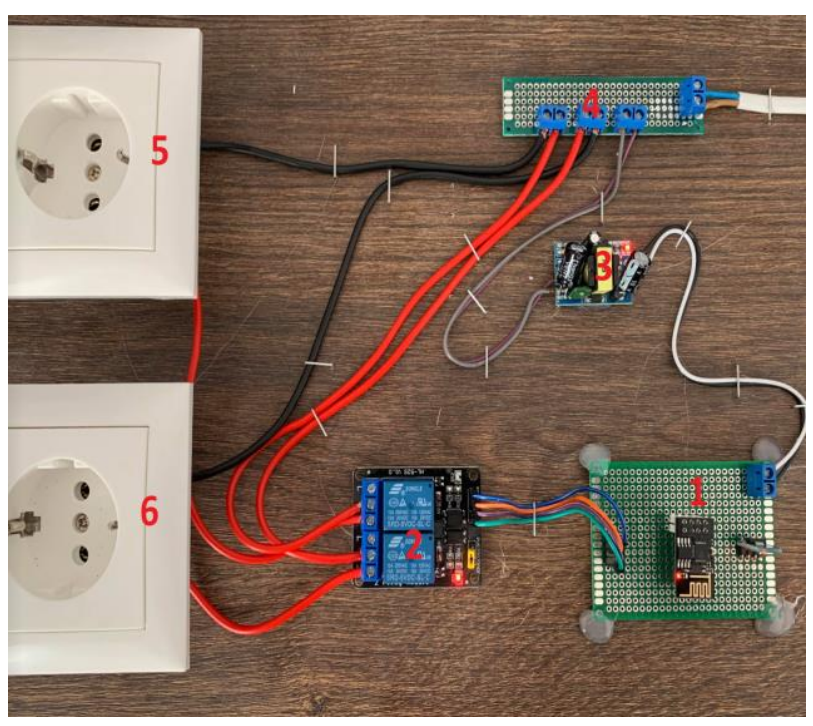

Figure 8. Electrical device control system.

\subsection{Software application}

The software application (Figure 10) is made for the ESP8266 module, the main programming language is Arduino IDE and for the web interface the HTML programming language is used. The control interface is made from 4 buttons, each device has an ON/OFF button. The web interface also shows the power state of each device individually.

\section{Electrical device control system}

\section{through Wi-Fi technology}

\section{Devices}

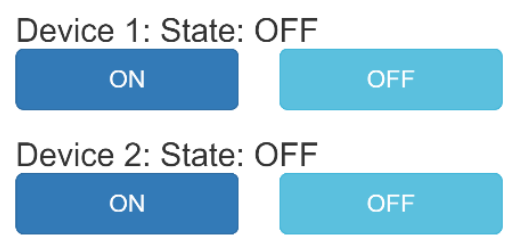

Figure 10. User web interface.

\section{CONCLUSIONS}

The system presented in this paper is a very useful to control various electronic devices via a Wi-Fi connection. The system can be controlled from anywhere using the default Ip and port. The control web page is easy to use, showing the status of each device. The connection is secure, requiring a username and password when logging in. The ESP-01 module is very versatile and economical and it supports various functions and sensors.

\section{REFERENCES}

[1] Agus Kurniawan, Internet of Things Projects with ESP32: Build Exciting and Powerful IoT Projects Using the All-new Espressif ESP32, 2019. 
[2] Forrest Mims, Getting Started in Electronics, Master Publishing, 1983.

[3] Charles Platt, Encyclopedia of Electronic Components Volume 1, Maker Media, 2012.

[4] John Boxall, Arduino Workshop, No Strach press, 2013.

[5] Jeremy Blum, Exploring Arduino: Tools and Techniques for Engineering Wizardry, John Wiley \& Sons, 2013. 